\title{
FLUXOGRAMA DE BENEFICIAMENTO PARA SEMENTES DE FEIJÃO-MUNGO-VERDE (Vigna radiata L.) ${ }^{1}$
}

\author{
ROBERTO FONTES ARAUJO ${ }^{2}$, EDUARDO FONTES ARAUJO ${ }^{3}$, JOÃO BATISTA ZONTA4 ${ }^{4}$, \\ ROGÉRIO FARIA VIEIRA², SÉRGIO MAURÍCIO LOPES DONZELES²
}

\begin{abstract}
RESUMO - O beneficiamento é componente fundamental em qualquer programa organizado de produção de sementes e visa aprimorar características do lote. Objetivou-se neste trabalho estabelecer um fluxograma de beneficiamento para sementes de feijão-mungo-verde (Vigna radiata L.). Utilizaram-se sementes dois lotes. O primeiro lote foi proveniente de colheita parcelada das vagens, em três épocas, e o segundo de colheita de toda a planta, com a maioria das vagens secas. Foram testados 13 fluxogramas de beneficiamento, com a utilização da máquina de ventilador e peneiras, separador pneumático, mesa de gravidade e classificador de peneiras. As sementes foram avaliadas quanto a sua qualidade física, fisiológica e sanitária. O fluxograma de beneficiamento ideal para lotes de sementes de feijão-mungo-verde provenientes de colheita parcelada das vagens é composto pela máquina de ventilador e peneiras, seguida da mesa de gravidade. Quando os lotes de sementes forem provenientes de colheita única das vagens o ideal é o uso da máquina de ventilador e peneiras, seguida do separador pneumático e da mesa de gravidade.
\end{abstract}

Termos para indexação: mesa de gravidade, massa específica, tamanho, qualidade fisiológica.

\section{PROCESSING FLOWCHART FOR MUNG BEAN (Vigna radiata L.) SEEDS}

\begin{abstract}
Seed processing is a key part of any organized seed production program and serves to improve seed lot quality. The objective of this study was to establish a processing flowchart for mung bean seeds (Vigna radiata $\mathrm{L}$.). Two seed lots were used: the first with seeds harvested at three different times and the second from a single harvest, with mostly mature pods. We tested 13 seed processing flowcharts, using a fan machine and sieves, a pneumatic separator, a gravity table and a sieve classifier. The seeds were evaluated for their physical, physiological and sanitary qualities. The ideal processing flowchart for mung bean seeds harvested at different times was the use of a fan machine and sieves followed by the gravity table. On the other hand, for seed lots from a single harvest, the ideal seed processing was a fan machine and sieves followed by a pneumatic separator and the gravity table.
\end{abstract}

Index terms: gravity table, size, specific mass, physiological quality.

${ }^{1}$ Submetido em 01/04/2010. Aceito para publicação em 12/11/2010.

${ }^{2}$ Eng. Agr, Dr., Pesquisador EPAMIG, 36570-000, Viçosa, MG. E-mail: rfaraujo@ufv.br, rfvieira@epamig.br, slopes@ufv.br
${ }^{3}$ Eng. Agr, Dr., Professor Associado III, Departamento de Fitotecnia, Universidade Federal de Viçosa, 36570-000, MG. E-mail: efaraujo@ufv.br

${ }^{4}$ Eng. Agr, Dr., Analista Embrapa Cocais, 65046-660, São Luís, MA. E-mail:*joao.zonta@embrapa.br 


\section{INTRODUÇÃO}

As sementes de feijão-mungo-verde ou mungo-verde (Vigna radiata L.) são utilizadas, principalmente, para a produção do broto de feijão (moyashi), forma de consumo muito apreciada na China, no Japão e em outros países. Quase desconhecida no Brasil até o início da década de 90, essa espécie tem, atualmente, um mercado relativamente grande no país (Vieira e Nishihara, 1992).

Muitos agricultores, principalmente em áreas irrigadas com agricultura mais intensiva, onde a mão-de-obra é mais escassa, têm realizado colheita única das plantas, com a maioria das vagens secas, em lugar de colher as vagens à medida que amadurecem. Como o feijão-mungo-verde é de hábito indeterminado, com maturação não uniforme, o uso desta prática, apesar de diminuir o custo de produção, reduz a qualidade das sementes e o potencial produtivo da cultura. Como o consumo do feijão-mungo-verde é feito principalmente na forma de brotos ("moyashi”), e não como grão, a qualidade das sementes é fundamental para o sucesso da produção dos brotos (Vieira e Nishihara, 1992).

Além do manejo correto da cultura e de cuidados rigorosos durante o processo de colheita, deve-se também dar atenção às etapas de processamento das sementes. $\mathrm{O}$ beneficiamento visa melhorar a qualidade do lote de sementes nos aspectos físico, fisiológico e sanitário, com a eliminação de impurezas, remoção de sementes de má qualidade e de outras espécies ou cultivares e classificação em frações mais uniformes (Carvalho e Nakagawa, 2000).

As máquinas utilizadas no beneficiamento baseiamse nas diferenças físicas existentes entre as sementes e os materiais indesejáveis (Vaughan et al., 1976; Gregg, 1983). Em geral, todos os lotes passam, primeiramente, pela máquina de ventiladores e peneiras, que têm como base de separação o tamanho e o peso específico, e é considerado equipamento básico na unidade de beneficiamento de sementes (Vaughan et al., 1976). A mesa de gravidade tem sido eficiente no aprimoramento dos lotes de diversas culturas; essa máquina elimina as sementes chochas, as atacadas por insetos ou microrganismos, outros tipos de impurezas, e classifica as sementes pelo seu peso específico (Gregg, 1983). O separador pneumático tem como base de separação o peso específico, eliminando impurezas e sementes mais leves do lote de sementes por meio de fluxo de ar (Peske, 2006). No classificador horizontal de peneiras as sementes são classificadas quanto à largura e espessura, sendo utilizado principalmente para milho e, atualmente para soja (Araújo et al., 2009).

A escolha e a sequência das máquinas a serem usadas no beneficiamento de sementes variam em função da espécie e das condições físicas do lote (Vaughan et al., 1976). Para algumas leguminosas comumente beneficiadas, já existem seqüências básicas. Para sementes de soja e ervilha, em geral, recomenda-se o uso da máquina de ventiladores e peneiras e mesa de gravidade ou máquina de ventiladores e peneiras, separador de espiral e mesa de gravidade; atualmente, algumas empresas estão usando o classificador de peneiras para classificar as sementes de soja pelo tamanho (Welch, 1980). Normalmente, os lotes de sementes de feijão-vagem e feijão comum são beneficiados pela máquina de ventiladores e peneiras e mesa de gravidade (Carvalho e Nakagawa, 2000 e Araújo et al., 2009). Para sementes de guandu, Ahrens e El Tassa (1999) afirmam que a utilização da mesa gravitacional, isoladamente ou em associação com a máquina de ventilador e peneiras, melhora a qualidade do lote de sementes nos aspectos físico e fisiológico e o uso exclusivo da máquina de ventilador e peneiras não interfere qualitativamente no lote beneficiado.

Sementes de soja (Baudet e Villela, 2007), ervilha (Araújo et al., 2009), feijão comum (Buitrago et al., 1991), feijão-vagem (Viggiano, 1990), ervilhaca-comum (Alexandre e Silva, 2000), e feijão-miúdo (Mertz et al., 2007) descarregadas na posição superior da mesa de gravidade apresentaram densidade, pesos unitário e volumétrico, poder germinativo, vigor, sanidade e pureza física maiores do que as descarregadas nas posições intermediárias e inferiores.

Os resultados de qualidade fisiológica de sementes em função do seu tamanho são contrastantes. Em alguns casos, sementes de soja maiores apresentaram maior germinação e lavouras mais produtivas (Baudet e Villela, 2007), e em outros, sementes pequenas de soja emergiram mais rapidamente e com raízes mais desenvolvidas (Sung, 1992). Ainda houve resultados que sementes de soja retidas nas peneiras intermediárias tiveram um maior potencial de armazenamento, com maior germinação e vigor (Santos et al., 2005), e outros, que o tamanho da semente de soja, para as cultivares estudadas, não afetou o desempenho da cultura no campo (Lima e Carmona, 1999 e Ávila et al., 2008).

Ressalta-se que há escassez de informações quanto à melhor forma de beneficiar sementes de feijão-mungoverde. Araújo et al. (2008) verificaram que o separador 
de espiral não foi eficiente na melhoria da qualidade dos lotes e a mesa de gravidade foi fundamental para o aumento da germinação, vigor e sanidade das sementes de feijão-mungo-verde. Constataram ainda que as sementes maiores, retidas na peneira superior do classificador horizontal, tiveram qualidade fisiológica inferior àquela das sementes menores, possivelmente por sofrerem maior dano mecânico no beneficiamento.

Objetivou-se no presente estudo estabelecer um fluxograma de beneficiamento para sementes de feijãomungo-verde (Vigna radiata L.).

\section{MATERIAL E MÉTODOS}

O trabalho foi conduzido na Unidade de Beneficiamento de Sementes (UBS) da Universidade Federal de Viçosa (UFV), no laboratório da Unidade Regional Epamig Zona da Mata (UREZM) da Empresa de Pesquisa Agropecuária de Minas Gerais (EPAMIG) e no Laboratório de Análise de Sementes do Departamento de Fitotecnia da UFV, em Viçosa - MG.

Foram utilizadas sementes de feijão-mungo-verde, cultivar Ouro Verde MG-2, provenientes de dois lotes de sementes produzidos simultaneamente na Fazenda Experimental do Vale do Piranga (FEVP), pertencente à EPAMIG, no município de Oratórios - MG. O primeiro lote foi proveniente de colheita parcelada das vagens, em três épocas (70, 80 e 90 dias após o plantio), e o segundo de colheita de toda a planta ( 80 dias após o plantio). Para ambos os lotes, a colheita das vagens procedeu-se de forma manual. As vagens foram levadas para um galpão ventilado, onde foram secadas à sombra e debulhadas com batidas de vara. Os lotes, compostos de sementes e impurezas (pedaços de vagem, galhos, folhas, torrões, pedras, terra etc.), foram secos à sombra, até o teor de água de aproximadamente $13 \%$.

Para estudar o efeito dos fluxogramas de beneficiamento foram usadas a máquina de ventilador e peneiras (MVP), o separador pneumático (SP), a mesa de gravidade (MG) e o classificador horizontal de peneiras planas (CP). Em alguns fluxogramas as sementes de peso intermediário, coletadas na bica 2 da $\mathrm{MG}$, foram repassadas na mesa com coleta final na bica 1. Na passagem das sementes pelo CP eliminaram-se as sementes muito grandes (retidas em peneira oblonga 3,9 x 18,3 mm), com risco de sofrerem danos mecânicos, e as muito pequenas (retidas em peneira oblonga 3,1 x 18,3 $\mathrm{mm}$ ), com pouquíssimo tecido de reserva, selecionando-se as sementes de tamanho intermediário (retidas em peneira oblonga 3,5 x 18,3 mm). Os lotes de sementes foram submetidos aos seguintes fluxogramas de beneficiamento:

1) $\mathrm{MVP} \rightarrow \mathrm{SP}$,

2) $\mathrm{MVP} \rightarrow \mathrm{SP} \rightarrow \mathrm{MG}$ (bica 1 ),

3) $\mathrm{MVP} \rightarrow \mathrm{SP} \rightarrow \mathrm{MG}$ (bica 2),

4) $\mathrm{MVP} \rightarrow \mathrm{SP} \rightarrow \mathrm{MG}$ (bica 2 ) $\rightarrow$ repasse na $\mathrm{MG}$ (bica 1),

5) $\mathrm{MVP} \rightarrow \mathrm{SP} \rightarrow \mathrm{MG}$ (bica 1$) \rightarrow \mathrm{CP}$,

6) $\mathrm{MVP} \rightarrow \mathrm{SP} \rightarrow \mathrm{MG}$ (bica 2 ) $\rightarrow$ repasse na $\mathrm{MG}$ (bica 1) $\rightarrow \mathrm{CP}$,

7) $\mathrm{MVP} \rightarrow \mathrm{SP} \rightarrow \mathrm{CP}$,

8) $\mathrm{MVP} \rightarrow \mathrm{SP} \rightarrow \mathrm{CP} \rightarrow \mathrm{MG}$ (bica 1 ),

9) $\mathrm{MVP} \rightarrow \mathrm{SP} \rightarrow \mathrm{CP} \rightarrow \mathrm{MG}$ (bica 2 ),

10) $\mathrm{MVP} \rightarrow \mathrm{SP} \rightarrow \mathrm{CP} \rightarrow \mathrm{MG}$ (bica 2 ) $\rightarrow$ repasse na $\mathrm{MG}$ (bica 1),

11) $\mathrm{MVP} \rightarrow \mathrm{MG}$ (bica 1 ),

12) $\mathrm{MVP} \rightarrow \mathrm{MG}$ (bica 2 e 3 ),

13) $\mathrm{MVP} \rightarrow \mathrm{MG}$ (bica 2 e 3 ) $\rightarrow$ repasse na MG (bica 1 ).

As avaliações da qualidade física e sanitária e a determinação do teor de água das sementes de cada amostra foram realizadas após a operação de beneficiamento (mês zero). Para determinação da qualidade fisiológica foram realizadas avaliações após o beneficiamento (mês zero) e aos 5 e 10 meses de armazenamento, sendo as sementes mantidas em embalagem de pano em sala sem controle de temperatura e umidade relativa, sendo a temperatura e a umidade relativa média registrada no período de duração do experimento de $22{ }^{\circ} \mathrm{C}$ e $80 \%$.

Determinação do teor de água (\%b.u.): empregouse o método da estufa, a $105+3{ }^{\circ} \mathrm{C}$, durante 24 horas, com duas repetições, conforme especificações das Regras para Análise de Sementes (Brasil, 1992); análise de pureza (\%): foram utilizadas quatro amostras de $100 \mathrm{~g}$ por tratamento. As sementes foram separadas em três frações (sementes puras, outras sementes e material inerte), conforme prescrito nas Regras para Análise de Sementes (Brasil, 1992); peso de mil sementes (g): feito de acordo com as Regras para Análise de Sementes (Brasil, 1992), utilizando-se oito repetições de 100 sementes; teste de germinação (\%): realizado com quatro repetições de 50 sementes, utilizando-se rolo de papel germitest (três folhas), umedecidas com água destilada na proporção de 2,5 vezes seu peso seco. Os rolos foram mantidos em germinador na temperatura de $25^{\circ} \mathrm{C}$. A avaliação foi feita no quinto e oitavo dias após a montagem e o resultado expresso em percentagem de plântulas normais (Brasil, 1992); teste de condutividade elétrica $\left(\mu \mathrm{S}^{-1} \mathrm{~cm}^{-1} \mathrm{~g}^{-1}\right)$ : realizado com 
quatro subamostras de 100 sementes por repetição. As 100 sementes, com massa conhecida, foram colocadas para embeber em copos plásticos com $75 \mathrm{~mL}$ de água destilada, a $25^{\circ} \mathrm{C}$, por 6 horas. Após agitação, foi feita a leitura em condutivímetro; teste de envelhecimento acelerado (\%): 200 sementes por tratamento foram colocadas em caixa gerbox, sobre tela, com $40 \mathrm{ml}$ de água destilada. O material foi acondicionado em BOD a $42{ }^{\circ} \mathrm{C}$, durante 72 horas (Marcos Filho, 1999). Em seguida, quatro subamostras de 50 sementes por tratamento foram submetidas ao teste de germinação; teste de sanidade: a detecção de fungos foi feita com o teste de papel de filtro modificado (bloter test), conforme metodologia descrita por Machado (1988). A identificação dos fungos presentes nas sementes foi realizada com auxílio de microscópio estereoscópico, expressando-se a percentagem de cada fungo detectado. Cada uma das quatro repetições constou de duas placas de polietileno com 25 sementes, totalizando 200 sementes por tratamento.

O delineamento experimental foi o inteiramente casualizado, num esquema fatorial 13 × 3 (13 fluxogramas de beneficiamento $\mathrm{x} 3$ períodos de avaliação), com quatro repetições. Para cada lote, os dados de pureza, peso de mil sementes, germinação e vigor foram submetidos à análise de variância e as médias dos tratamentos comparadas pelo teste de Skott-Knott, a 5\% de probabilidade.

\section{RESULTADOS E DISCUSSÃO}

Pela análise de variância, observou-se que não houve efeito significativo para a interação fluxogramas de beneficiamento versus período de armazenamento e nem para os períodos de armazenamento, sendo observado efeito significativo somente para os fluxogramas de beneficiamento. Sendo assim, os valores relativos aos diferentes fluxogramas de beneficiamento, para os testes de avaliação da qualidade fisiológica, foram apresentados com as médias obtidas com os valores das três avaliações (zero, cinco e dez meses).

Ressalta-se, inicialmente, que os tratamentos 3, 9 e 12, cujas amostras de sementes foram retiradas da bica 2 da mesa de gravidade, não representam o fim de uma linha de beneficiamento. Foram utilizados para facilitar a interpretação e a discussão dos resultados.

O teor de água das sementes dos diferentes lotes e tratamentos, após a secagem, era de, aproximadamente, $12 \%$, portanto, adequado para o beneficiamento e o armazenamento das sementes (Tabela 1).

TABELA 1. Características físicas de lotes de sementes de feijão-mungo-verde depois de submetidos a diferentes fluxogramas de beneficiamento.

\begin{tabular}{ccccccc}
\hline & \multicolumn{3}{c}{ Lote 1 (Colheita parcelada) } & \multicolumn{3}{c}{ Lote 2 (Colheita única) } \\
\cline { 2 - 6 } Fluxogramas & $\begin{array}{c}\text { Teor de água } \\
(\%)\end{array}$ & $\begin{array}{c}\text { Pureza } \\
(\%)\end{array}$ & $\begin{array}{c}\text { Peso de mil } \\
\text { sementes }(\mathrm{g})\end{array}$ & $\begin{array}{c}\text { Teor de água } \\
(\%)\end{array}$ & $\begin{array}{c}\text { Pureza }(\%) \\
\text { Peso de mil } \\
\text { sementes }(\mathrm{g})\end{array}$ \\
\hline 1 & 12,5 & $99 \mathrm{a}$ & $41,7 \mathrm{~b}$ & 12,2 & $99 \mathrm{a}$ & $39,3 \mathrm{~b}$ \\
2 & 11,9 & $99 \mathrm{a}$ & $44,6 \mathrm{a}$ & 12,5 & $99 \mathrm{a}$ & $42,8 \mathrm{a}$ \\
3 & 12,3 & $99 \mathrm{a}$ & $38,7 \mathrm{c}$ & 12,5 & $98 \mathrm{a}$ & $36,5 \mathrm{c}$ \\
4 & 12,4 & $99 \mathrm{a}$ & $41,2 \mathrm{~b}$ & 12,4 & $98 \mathrm{~b}$ & $36,9 \mathrm{c}$ \\
5 & 12,2 & $99 \mathrm{a}$ & $44,7 \mathrm{a}$ & 12,3 & $99 \mathrm{a}$ & $42,6 \mathrm{a}$ \\
6 & 11,9 & $99 \mathrm{a}$ & $40,9 \mathrm{~b}$ & 12,1 & $98 \mathrm{a}$ & $37,3 \mathrm{c}$ \\
7 & 12,3 & $99 \mathrm{a}$ & $41,9 \mathrm{~b}$ & 11,9 & $99 \mathrm{a}$ & $40,3 \mathrm{~b}$ \\
8 & 12,4 & $99 \mathrm{a}$ & $44,3 \mathrm{a}$ & 12,2 & $99 \mathrm{a}$ & $42,9 \mathrm{a}$ \\
9 & 12,4 & $99 \mathrm{a}$ & $38,9 \mathrm{c}$ & 12,4 & $98 \mathrm{a}$ & $37,3 \mathrm{c}$ \\
10 & 12,2 & $99 \mathrm{a}$ & $41,4 \mathrm{~b}$ & 12,3 & $98 \mathrm{a}$ & $39,4 \mathrm{~b}$ \\
11 & 12,2 & $99 \mathrm{a}$ & $43,8 \mathrm{a}$ & 12,3 & $99 \mathrm{a}$ & $39,9 \mathrm{~b}$ \\
12 & 12,4 & $96 \mathrm{~b}$ & $36,9 \mathrm{~d}$ & 12,2 & $89 \mathrm{~b}$ & $34,8 \mathrm{~d}$ \\
\hline
\end{tabular}

Médias seguidas da mesma letra na coluna pertencem ao mesmo grupo pelo Teste de Skott-Knott, a 5\% de probabilidade. 
Pelos resultados de análise de pureza do tratamento 12 , no qual as sementes não passaram pela limpeza no separador pneumático e foram retiradas da bica 2 da mesa de gravidade, verifica-se que o lote 2 ( $89 \%$ de pureza) se apresentava com quantidade de impureza bem superior ao lote 1 (96\% de pureza) (Tabela 1). A razão para essa diferença é que o lote 1 foi obtido a partir de colheita parcelada das vagens e o lote 2 de colheita única, da planta toda; consequentemente, uma maior quantidade de impurezas (pedaços de folha, pedaços de galhos, torrões de terra e outras) acompanhou as vagens colhidas do lote 2. Em ambos os lotes, nos tratamentos de beneficiamento em que as sementes passaram pelo separador pneumático, mesmo naqueles envolvendo amostras de sementes provenientes da bica 2 da mesa de gravidade, não houve diferenças significativas entre as médias de pureza, demonstrando a eficiência do equipamento em separar as sementes das impurezas mais leves.

Quanto ao peso de mil sementes do lote 1, verifica-se que as sementes coletadas na bica 1 da mesa de gravidade, sem repasse (tratamentos 2, 5, 8 e 11), apresentaram valores significativamente superiores àquelas dos demais tratamentos, demonstrando a eficiência da máquina para classificar as sementes de feijão-mungo-verde (Tabela 1). Resultados semelhantes foram encontrados por Buitrago et al. (1991), os quais verificaram que sementes de feijão, beneficiadas na máquina de ventilador e peneiras, seguida da mesa de gravidade, ou apenas na mesa de gravidade, descarregadas nas partes intermediária e baixa, apresentaram peso de mil sementes inferior às sementes da parte alta (bica 1). Com relação ao lote 2, os resultados foram semelhantes aos do lote 1 em termos de eficiência das máquinas. Entretanto, o tratamento 11 proporcionou peso de mil sementes inferior aos tratamentos 2,5 e 8 ; esses resultados podem ser explicados pelo fato de o lote 2 ter sido colhido com mais impurezas e as sementes do tratamento em questão não terem passado pelo separador pneumático para o término da limpeza.

A germinação ao longo do armazenamento, para todos os tratamentos, nos dois lotes, praticamente não se alterou, demonstrando ser o feijão-mungo-verde uma espécie que conserva bem a qualidade fisiológica das sementes (Tabela 2). Verifica-se que em todos os tratamentos compostos por amostras retiradas da bica 1 da mesa de gravidade (tratamentos 2, 5, 8 e 11) as sementes tiveram a sua germinação significativamente superior às dos demais tratamentos; neste caso, é evidente a eficiência da mesa de gravidade, pois no tratamento 11 , as sementes não passaram pelo separador pneumático. A importância da mesa de gravidade é confirmada em estudo realizado por Araujo et al. (2008), os quais consideram a máquina fundamental na linha de beneficiamento de sementes desta leguminosa. Ressalta-se que o repasse na mesa de gravidade das sementes da bica 2 não melhorou a germinação das sementes (tratamentos 4, 6, 10 e 13). Com relação ao lote dois, os resultados de germinação diferem daqueles do lote 1 apenas no tratamento 11, que não passou pelo separador pneumático, apresentando germinação significativamente inferior aos tratamentos 2,5 e 8 . Isto sugere que lotes com menos impurezas, obtidos a partir de colheita parcelada das vagens, podem ser beneficiados apenas pela máquina de ventilador e peneiras, para a limpeza, seguida da mesa de gravidade para classificação das sementes pelo peso específico. Já os lotes com mais impurezas (pedaços de folha, pedaços de galhos, torrões de terra, etc), provenientes de colheita única das vagens, devem passar pela máquina de ventilados e peneiras, seguidos do separador pneumático, para terminar a limpeza, finalizando com a mesa de gravidade, para classificar as sementes pelo peso específico. Esses resultados com feijão-mungo-verde são semelhantes aos encontrados com soja (Baudet e Villela, 2007), ervilha (Nascimento, 1994), feijão comum (Buitrago et al., 1991), feijão-vagem (Viggiano, 1990) e feijão-miúdo (Mertz et al., 2007). Esses autores verificaram que as sementes descarregadas nas posições superiores da mesa de gravidade apresentaram densidade, pesos unitário e volumétrico, poder germinativo, vigor, sanidade e pureza física maiores que os daquelas descarregadas nas posições intermediárias e inferiores.

Os resultados de vigor obtidos no teste de envelhecimento acelerado (Tabela 2), no teste de frio (Tabela 3) e no teste de condutividade elétrica (Tabela 3) indicam a mesma tendência daqueles de germinação; sementes do lote 1 , provenientes de colheita parcelada das vagens, tiveram maior vigor nos tratamentos $2,5,8 \mathrm{e}$ 11 , e sementes do lote 2 , provenientes de colheita única das vagens, tiveram o vigor no tratamento 11 menor, em relação, principalmente, aos tratamentos 2 e 5 .

Com relação à qualidade sanitária das sementes, foram identificados nas sementes os fungos Alternaria $s p$, Colletotrichum dematium e Fusarium spp., com uma maior incidência da última espécie (Tabela 4). Houve forte correlação negativa da incidência de fungos com a germinação e o vigor das sementes. De maneira geral, as sementes provenientes de plantas colhidas com a maioria das vagens maduras (lote 2) 
apresentaram menor vigor e maior incidência de fungos que as originadas da colheita das vagens à medida que amadureciam. Provavelmente, a maior permanência de algumas vagens do lote 2 no campo, após a maturidade fisiológica das sementes, reduziu-lhes a qualidade fisiológica e sanitária. Os resultados foram semelhantes para os tratamentos de beneficiamento. Aqueles que tiveram sementes com germinação e o vigor menores, foram os mesmos cujas sementes apresentaram maior incidência de fungos. Sendo assim, vale ressaltar a importância da mesa de gravidade nos tratamentos 2, 5, 8 e 11 , em que as sementes de maior densidade apresentaram menor incidência de fungos e maiores germinação e vigor. Mertz et al. (2007) observaram que a atuação da mesa gravitacional permite a remoção de frações de sementes com menor densidade e promove alterações positivas à qualidade fisiológica e sanitária dos lotes de sementes de feijão-miúdo.

TABELA 2. Teste de germinação (TG) e de envelhecimento acelerado (EA) de lotes de sementes de feijão-mungoverde, depois de submetidos a diferentes fluxogramas de beneficiamento.

\begin{tabular}{ccccc}
\hline \multirow{2}{*}{ Fluxogramas } & \multicolumn{2}{c}{ Lote 1 (Colheita parcelada) } & \multicolumn{2}{c}{ Lote 2(Colheita única) } \\
\cline { 2 - 5 } & $\mathrm{G}(\%)$ & EA $(\%)$ & $90 \mathrm{~b}(\%)$ \\
\hline 1 & $91 \mathrm{~b}$ & $81 \mathrm{~b}$ & $97 \mathrm{a}$ & $76 \mathrm{c}$ \\
2 & $96 \mathrm{a}$ & $90 \mathrm{a}$ & $84 \mathrm{c}$ & $62 \mathrm{f}$ \\
3 & $85 \mathrm{c}$ & $70 \mathrm{~d}$ & $89 \mathrm{~b}$ & $71 \mathrm{e}$ \\
4 & $90 \mathrm{~b}$ & $75 \mathrm{c}$ & $97 \mathrm{a}$ & $89 \mathrm{a}$ \\
5 & $97 \mathrm{a}$ & $89 \mathrm{a}$ & $89 \mathrm{~b}$ & $72 \mathrm{~d}$ \\
6 & $91 \mathrm{~b}$ & $76 \mathrm{c}$ & $90 \mathrm{~b}$ & $78 \mathrm{c}$ \\
7 & $92 \mathrm{~b}$ & $82 \mathrm{~b}$ & $97 \mathrm{a}$ & $84 \mathrm{~b}$ \\
8 & $96 \mathrm{a}$ & $89 \mathrm{~b}$ & $67 \mathrm{e}$ \\
9 & $90 \mathrm{~b}$ & $88 \mathrm{a}$ & $91 \mathrm{~b}$ & $89 \mathrm{e}$ \\
10 & $91 \mathrm{~b}$ & $69 \mathrm{~d}$ & $91 \mathrm{~b}$ & $81 \mathrm{~b}$ \\
11 & $96 \mathrm{a}$ & $76 \mathrm{c}$ & $83 \mathrm{c}$ & $68 \mathrm{e}$ \\
\hline
\end{tabular}

Médias seguidas da mesma letra na coluna pertencem ao mesmo grupo pelo Teste de Skott-Knott, a 5\% de probabilidade.

TABELA 3. Teste de frio (TF) e de condutividade elétrica (CE) de lotes de sementes de feijão-mungo-verde, depois de submetidos a diferentes fluxogramas de beneficiamento.

\begin{tabular}{|c|c|c|c|c|}
\hline \multirow{2}{*}{ Fluxogramas } & \multicolumn{2}{|c|}{ Lote 1 (Colheita parcelada) } & \multicolumn{2}{|c|}{ Lote 2 (Colheita única) } \\
\hline & $\mathrm{TF}(\%)$ & $\mathrm{CE}\left(\mu \mathrm{S}^{-1} \mathrm{~cm}^{-1} \mathrm{~g}^{-1}\right)$ & $\mathrm{TF}(\%)$ & $\mathrm{CE}\left(\mu \mathrm{S}^{-1} \mathrm{~cm}^{-1} \mathrm{~g}^{-1}\right)$ \\
\hline 1 & $90 \mathrm{~b}$ & $57,58 \mathrm{c}$ & $90 \mathrm{~b}$ & $63,03 \mathrm{c}$ \\
\hline 2 & $96 \mathrm{a}$ & $51,13 \mathrm{~d}$ & $95 \mathrm{a}$ & $51,75 \mathrm{e}$ \\
\hline 3 & $83 \mathrm{c}$ & $65,46 \mathrm{~b}$ & $82 \mathrm{~d}$ & $70,05 \mathrm{~b}$ \\
\hline 4 & $87 \mathrm{c}$ & $64,66 \mathrm{~b}$ & $86 \mathrm{c}$ & $63,31 \mathrm{c}$ \\
\hline 5 & $97 \mathrm{a}$ & $50,96 \mathrm{~d}$ & $96 \mathrm{a}$ & $51,63 \mathrm{e}$ \\
\hline 6 & $91 \mathrm{~b}$ & $63,86 \mathrm{~b}$ & $89 \mathrm{~b}$ & $63,61 \mathrm{c}$ \\
\hline 7 & $91 \mathrm{~b}$ & $53,95 \mathrm{c}$ & $91 \mathrm{~b}$ & $57,68 \mathrm{~d}$ \\
\hline 8 & $97 \mathrm{a}$ & $50,17 \mathrm{~d}$ & $95 \mathrm{a}$ & $51,45 \mathrm{e}$ \\
\hline 9 & $83 \mathrm{c}$ & $65,51 \mathrm{~b}$ & $82 \mathrm{~d}$ & $70,82 \mathrm{~b}$ \\
\hline 10 & $90 \mathrm{~b}$ & $58,14 \mathrm{c}$ & $86 \mathrm{c}$ & $63,13 \mathrm{c}$ \\
\hline 11 & $96 \mathrm{a}$ & $51,58 \mathrm{~d}$ & $92 \mathrm{a}$ & $58,24 \mathrm{~d}$ \\
\hline 12 & $80 \mathrm{~d}$ & $72,50 \mathrm{a}$ & $80 \mathrm{~d}$ & $77,75 \mathrm{a}$ \\
\hline 13 & $86 \mathrm{c}$ & $64,88 \mathrm{~b}$ & $85 \mathrm{c}$ & $68,18 \mathrm{~b}$ \\
\hline
\end{tabular}

Médias seguidas da mesma letra na coluna pertencem ao mesmo grupo pelo Teste de Skott-Knott, a 5\% de probabilidade. 
TABELA 4. Incidência de fungos (\%) em lotes de sementes de feijão-mungo-verde, após submetidos a diferentes fluxogramas de beneficiamento.

\begin{tabular}{|c|c|c|c|c|c|c|}
\hline \multirow[b]{2}{*}{ Fluxogramas } & \multicolumn{3}{|c|}{ Lote 1 (Colheita parcelada) } & \multicolumn{3}{|c|}{ Lote 2 (Colheita única) } \\
\hline & $\begin{array}{c}\text { Alternaria } \\
s p .\end{array}$ & C. dematium ${ }^{1}$ & Fusarium spp. & Alternaria sp. & C. dematium & $\begin{array}{c}\text { Fusarium } \\
\text { spp. }\end{array}$ \\
\hline 1 & 2 & 1 & 3 & 4 & 2 & 5 \\
\hline 2 & 0 & 0 & 0 & 0 & 0 & 1 \\
\hline 3 & 4 & 4 & 6 & 5 & 7 & 9 \\
\hline 4 & 2 & 2 & 2 & 3 & 4 & 3 \\
\hline 5 & 0 & 0 & 0 & 0 & 1 & 0 \\
\hline 6 & 1 & 2 & 2 & 2 & 2 & 3 \\
\hline 7 & 1 & 1 & 1 & 2 & 2 & 3 \\
\hline 8 & 0 & 0 & 0 & 0 & 0 & 0 \\
\hline 9 & 2 & 3 & 3 & 4 & 6 & 6 \\
\hline 10 & 1 & 1 & 2 & 3 & 3 & 5 \\
\hline 11 & 0 & 0 & 1 & 2 & 1 & 2 \\
\hline 12 & 6 & 5 & 8 & 8 & 8 & 11 \\
\hline 13 & 2 & 2 & 2 & 4 & 5 & 5 \\
\hline
\end{tabular}

${ }^{1}$ Colletotrichum dematium

\section{CONCLUSÕES}

O fluxograma de beneficiamento ideal, para lotes de sementes de feijão-mungo-verde provenientes de campo onde a colheita das vagens for parcelada, é composto pela máquina de ventilador e peneiras, seguida da mesa de gravidade;

Quando os lotes de sementes de feijão-mungo-verde forem provenientes de colheita única das vagens, ou seja, de toda planta, o ideal é o uso da máquina de ventilador e peneiras, seguida do separador pneumático, finalizando com a mesa de gravidade.

\section{AGRADECIMENTOS}

À Fundação de Amparo à Pesquisa do Estado de Minas Gerais (FAPEMIG), pelo patrocínio desse projeto de pesquisa e pela concessão da bolsa de pesquisa ao primeiro autor.

\section{REFERÊNCIAS}

AHRENS, D.C.; EL TASSA, S.O.M. Avaliações do beneficiamento de sementes de guandu (Cajanus cajan (L.) Millsp.). Revista Brasileira de Sementes, v.21, n.1, p.27-
31, 1999. http://www.abrates.org.br/revista/artigos/1999/ v21n1/artigo04.pdf

ALEXANDRE, A.D.; SILVA, W.R. Mesa gravitacional e qualidade física de sementes de ervilhaca-comum (Vicia sativa L.). Revista Brasileira de Sementes, v.22, n.2, p.223-230, 2000. http://www.abrates.org.br/revista/ artigos/2000/v22n2/artigo30.pdf

ARAÚJO, E.F.; VIGGIANO, J.; SILVA, R.F. Beneficiamento de sementes de hortaliças. In: NASCIMENTO, W.M. (Ed.). Tecnologia de sementes de hortaliças. Brasília: Embrapa Hortaliças, 2009. p.105134.

ARAÚJO, R.F.; ARAUJO, E.F.; VIEIRA, R.F.; SOFIATTI, V.; ZONTA, J.B.; SOUZA, L.T. Qualidades fisiológica e sanitária de sementes de feijão-mungo-verde submetidas ao beneficiamento. Revista Brasileira de Armazenamento, v.33, n.1, p.43-51, 2008.

ÁVILA, W.; PERIN, A.; GUARESCHI, R.F.; GAZOLA, P.R. Influência do tamanho da semente na produtividade de variedades de soja. Agrarian, v.1, n.2, p.83-89, 2008. http://www.periodicos.ufgd.edu.br/index.php/agrarian/ article/viewFile/257/209

BAUDET, L.; VILLELA, F.A. Unidades de beneficiamento de sementes. Seed News, v.11, n.2, p.22-26, 2007. 
BRASIL. Ministério da Agricultura e Reforma Agrária. Secretaria Nacional de Defesa Agropecuária. Departamento Nacional de Produção Vegetal. Coordenação de Laboratório Vegetal. Regras para Análise de Sementes. Brasília, DF, 1992. 365p.

BUITRAGO, I.C.; VILLELA, F.A.; TILLMANN, M.A.A.; da SILVA, J.B. Perdas e qualidade de sementes de feijão beneficiadas em máquina de ventiladores e peneiras e mesa de gravidade. Revista Brasileira de Sementes, v.13, n.2, p.99-104, 1991. http://www.abrates.org.br/revista/ artigos/1991/v13n2/artigo04.pdf

CARVALHO, N.M.; NAKAGAWA, J. Sementes: ciência, tecnologia e produção. 4.ed. Jaboticabal: FUNEP, 2000. $588 \mathrm{p}$.

GREGG, B.R. Seed processing in the tropics. Seed Science and Technology, v.11, n.1, p.19-39, 1983.

LIMA, A.M.M.P.; CARMONA, R. Influência do tamanho da semente no desempenho produtivo da soja. Revista Brasileira de Sementes, v.21, n.1, p.157-163, 1999. http:// www.abrates.org.br/revista/artigos/1999/v21n1/artigo24. pdf

MACHADO, J.C. Patologia de sementes: fundamentos e aplicações. Lavras: ESAL/FAESP, 1988. 107p.

MARCOS FILHO, J. Teste de envelhecimento acelerado. In: KRZYZANOWSKI, F.C.; VIEIRA, R.D.; FRANÇA NETO, J.B. (Ed.). Vigor de sementes: conceitos e testes. Londrina: Associação Brasileira de Tecnologia de Sementes, 1999. Cap.3, p.1-24.

MERTZ, L.M.; HENNING, F.A.; MAIA, M.S.; MENEGHELlO, G.E.; HENRIQUES, A.; MADAIL, R. Qualidade fisiológica e sanitária de sementes de feijãomiúdo beneficiadas em mesa gravitacional. Revista
Brasileira de Sementes, v.29, n.3, p.1-7, 2007. http:// www.scielo.br/pdf/rbs/v29n3/a01v29n3.pdf

NASCIMENTO, W.M. Efeito do beneficiamento na qualidade de semente de ervilha. Pesquisa Agropecuária Brasileira, v.29, n.2, p.309-313, 1994.

PESKE, S.T. Sementes: fundamentos científicos e tecnológicos. 2.ed. Pelotas: Universitária, 2006. 470p. http://webnotes.sct.embrapa.br/pdf/pab1994/fevereiro/ pab18_fev_94.pdf

SANTOS, P.M.; REIS, M.S.; SEDIYAMA, T.; ARAUJO, E.F.; CECON, P.R.; SANTOS, M.R. feito da classificação por tamanho da semente de soja na sua qualidade fisiológica durante o armazenamento. Acta Scientiarum Agronomy, v.27, n.3, p.395-402, 2005. http://periodicos.uem.br/ojs/ index.php/ActaSciAgron/article/view/1398/814

SUNG, F.J.M. Field emergence of edible soybean seeds differing in seed size and emergence strength. Seed Science and Technology, v.20, n.3, p.527-532, 1992.

VAUGHAN, C.E.; GREGG，E.R.; DELOUCHE，J.C. Beneficiamento e manuseio de sementes. Brasil, Ministério da Agricultura/AGIPLAN, 1976.

VIEIRA, R.F.; NISHIHARA, M.K. Comportamento de cultivares de mungo-verde (Vigna radiata) em Viçosa, Minas Gerais. Revista Ceres, v.39, n.221, p.60-83, 1992.

VIGGIANO, J. Produção de sementes de feijão-vagem. In: CASTELLANE, P.D.; NICOLOSI, W.M.; HASEGAWA, M. (Ed.). Produção de sementes de hortaliças. Jaboticabal: FCAV: FUNEP, 1990. p.127-140.

WELCH, G.B. Beneficiamento de sementes no Brasil. Brasília, DF: SNAP/CSM, 1980. 205p. 